\title{
SCATTERING THEORY FOR SEMILINEAR WAVE EQUATIONS WITH SMALL DATA IN TWO SPACE DIMENSIONS
}

\author{
KIMITOSHI TSUTAYA
}

\begin{abstract}
We study scattering theory for the semilinear wave equation $u_{t t}-$ $\Delta u=|u|^{p-1} u$ in two space dimensions. We show that if $p>p_{0}=(3+\sqrt{17}) / 2$, the scattering operator exists for smooth and small data. The lower bound $p_{0}$ of $p$ is considered to be optimal (see Glassey [6, 7], Schaeffer [18]). Our result is an extension of the results by Strauss [19], Klainerman [10], and Mochizuki and Motai $[14,15]$. The construction of the scattering operator for small data does not follow directly from the proofs in [7, 13, 20 and 22] concerning the global existence of solutions for the Cauchy problem of the above equation with small initial data given at $t=0$ in two space dimensions, because we have to consider the integral equation with unbounded integral region associated to the above equation:

$$
u(x, t)=u_{0}^{-}(x, t)+\frac{1}{2 \pi} \int_{-\infty}^{t} \int_{|x-y| \leq t-s} \frac{\left(|u|^{p-1} u\right)(y, s)}{\sqrt{(t-s)^{2}-|x-y|^{2}}} d y d s,
$$

for $t \in R$, where $u_{0}^{-}(x, t)$ is a solution of $u_{t t}-\Delta u=0$ which $u(x, t)$ approaches asymptotically as $t \rightarrow-\infty$. The proof of the basic estimate for the above integral equation is more difficult and complicated than that for the Cauchy problem of $u_{t t}-\Delta u=|u|^{p-1} u$ in two space dimensions.
\end{abstract}

\section{INTRODUCTION}

We consider a scattering problem for the semilinear wave equation

$$
u_{t t}-\Delta u=F(u), \quad(x, t) \in R^{n} \times R,
$$

where $F(u)=\lambda|u|^{p}$ or $\lambda|u|^{p-1} u, \lambda \in R, p>1$, and $n=2$.

We compare the asymptotic behavior as $t \rightarrow \pm \infty$ of solution of (1.1) with those of suitable solutions of the Cauchy problem for the free wave equation

$$
u_{t t}-\Delta u=0, \quad(x, t) \in R^{n} \times R,
$$

in the sense of energy norm.

Among many theorems on the construction of the scattering operator for (1.1), we mention a remarkable result of Pecher [16] which has improved previous results by Strauss [19], Klainerman [10], and Mochizuki and Motai [14, $15]$.

Pecher has shown that in three space dimensions $(n=3)$, the scattering operator for (1.1) exists for regular and small data if $p>1+\sqrt{2}$. This lower bound

Received by the editors February 17, 1992.

1991 Mathematics Subject Classification. Primary 35P25, 35L70.

Key words and phrases. Scattering theory, semilinear wave equations, two space dimensions. 
of $p$ is optimal, since John [8] showed that there exist global $C^{2}$-solutions to the Cauchy problem for the equation $u_{t t}-\Delta u=|u|^{p}$ with smooth and small data of compact support if $p>1+\sqrt{2}$, and it was also shown by John $[8,9]$ and Schaeffer [18] that the solution blows up in finite time for small data of compact support if $1<p \leq 1+\sqrt{2}$.

In two space dimensions, Strauss [19] proved that the scattering operator exists if $p>2+\sqrt{5}$, provided the smooth data are small. This result was improved to $p>2+\sqrt{3}$ by Klainerman [10] and Mochizuki and Motai [14, 15]. However, these results are not sharp, since Glassey [7] proved that the Cauchy problem for $u_{t t}-\Delta u=|u|^{p}$ has global $C^{2}$-solutions if $p>(3+\sqrt{17}) / 2$, provided the smooth data of compact support are small, and moreover it was shown by Glassey [6] and Schaeffer [18] that if $1<p \leq(3+\sqrt{17}) / 2$, global solutions do not exist, provided the data of compact support satisfy a certain condition.

Our aim of this paper is to prove that if $p>(3+\sqrt{17}) / 2$, the scattering operator exists for smooth and small data in two space dimensions.

We note that in the global existence theorems by John and Glassey, the data are compactly supported. Since we solve the Cauchy problem for (1.1) with the data given at $t=-\infty$, the assumption of the compactly supported data is not natural. In this connection, we briefly state the previous results concerning the Cauchy problem

$$
\begin{gathered}
u_{t t}-\Delta u=|u|^{p}, \quad(x, t) \in R^{n} \times(0, \infty), \\
u(x, 0)=f(x), \quad u_{t}(x, 0)=g(x), \quad x \in R^{n}(n=2,3),
\end{gathered}
$$

with small data of noncompact support.

Asakura [2], Kubota [13], and the author [20, 21, 22] have shown that global solutions exist for small data $f(x) \in C^{3}\left(R^{n}\right), g(x) \in C^{2}\left(R^{n}\right)$ satisfying

$$
\mathrm{D}_{x}^{\alpha} f(x), \mathrm{D}_{x}^{\beta} g(x)=O\left(|x|^{-1-k}\right) \text { as }|x| \rightarrow \infty,|\alpha| \leq 3,|\beta| \leq 2,
$$

if $k \geq 2 /(p-1), p>1+\sqrt{2}$ for $n=3$, and $p>(3+\sqrt{17}) / 2$ for $n=2$. The result due to Pecher [16] also corresponds to the removal of compact support assumption for initial data in three space dimensions.

In contrast to this existence theorem, Asakura [2], Agemi and Takamura [1], and the author [20] have proved that if the data satisfy

$$
f(x)=0, \quad g(x) \geq \frac{\varepsilon}{(1+|x|)^{1+k}}, \quad \varepsilon>0,
$$

and $0<k<2 /(p-1)$, then the solution blows up in finite time even with

$$
p> \begin{cases}1+\sqrt{2} & (n=3), \\ (3+\sqrt{17}) / 2 & (n=2) .\end{cases}
$$

The construction of the scattering operator for small data is almost equivalent to the construction of the global solution for the Cauchy problem of (1.1) with small initial data given at $t=0$ in the case of three space dimensions, but the construction of the scattering operator for small data does not follow directly from the proof of global existence of solutions for the Cauchy problem of (1.1) with small initial data given at $t=0$ in the case of two space 
dimensions, because we have to consider the integral equation with unbounded integral region associated to (1.1):

$$
u(x, t)=u_{0}^{-}(x, t)+\frac{1}{2 \pi} \int_{-\infty}^{t} \int_{|x-y| \leq t-s} \frac{F(u(y, s))}{\sqrt{(t-s)^{2}-|x-y|^{2}}} d y d s,
$$

for $t \in R$,

where $u_{0}^{-}(x, t)$ is a solution of (1.2) which $u(x, t)$ approaches asymptotically as $t \rightarrow-\infty$. The integral of the right-hand side consists of the integral on $(-\infty, 0]$ and the integral on $[0, t]$ for $t>0$ or $[t, 0]$ for $t<0$. The integral on $[0, t], t>0$, or on $[t, 0], t<0$, is the same as the integral appearing in the Cauchy problem of $(1.1)$ at $t=0$. In the case of three space dimensions, the evaluation of the integral on $(-\infty, 0]$ is quite the same as that of the integral on $[0, t], t>0$, or $[t, 0], t<0$. But in the case of two space dimensions, we cannot evaluate the integral on $(-\infty, 0]$ in the same way as the integral on $[0, t], t>0$, or $[t, 0], t<0$, due to the speciality of two space dimensions. We have to split the integral region into several parts and to make proper use of all the estimates $(4.7 \mathrm{a})-(4.8 \mathrm{~b})$ in $\S 4$ for the integral kernel in each part of the integral region. Such a procedure is not needed in three space dimensions. For the Cauchy problem at $t=0$ in two space dimensions, (4.7b) and (4.8b) are sufficient and so we do not have to use (4.7a) and (4.8a) (see [13, 20, 22]). Accordingly, the proof of the basic estimate (see Lemma 4.1) for (1.4) is more difficult and complicated than that for the Cauchy problem of $(1.3)$ in two space dimensions.

For the case of large data, we refer to the results by Ginibre and Velo $[3,4$, 5].

\section{ASSUMPTIONS AND THEOREM}

We study the scattering problem for the semilinear wave equation of the form

$$
u_{t t}-\Delta u=F(u), \quad(x, t) \in R^{2} \times R .
$$

We make the following hypothesis.

(H1) $F(u) \in C^{2}(R)$ and there exist $p>(3+\sqrt{17}) / 2, \lambda>0$, such that

$$
\begin{gathered}
\left|F^{(j)}(u)\right| \leq \lambda|u|^{p-j} \quad \text { for }|u| \leq 1, j=0,1,2, \\
\left|F^{\prime \prime}(u)-F^{\prime \prime}(v)\right| \leq \lambda|\phi|^{p-3}|u-v| \text { for }|u|,|v| \leq 1, \phi=\max \{|u|,|v|\} .
\end{gathered}
$$

Let $u_{0}^{-}(x, t)$ be the $C^{2}$-solution of the Cauchy problem

$$
\begin{gathered}
u_{t t}-\Delta u=0, \quad(x, t) \in R^{2} \times R, \\
u(x, 0)=f(x), \quad u_{t}(x, 0)=g(x), \quad x \in R^{2} .
\end{gathered}
$$

The hypothesis concerning the data is the following (H2) $f(x) \in C^{3}\left(R^{2}\right), g(x) \in C^{2}\left(R^{2}\right)$ satisfy

$$
\sum_{|\alpha| \leq 3}\left|\mathrm{D}_{x}^{\alpha} f(x)\right|+\sum_{|\beta| \leq 2}\left|\mathrm{D}_{x}^{\beta} g(x)\right| \leq \frac{\varepsilon}{(1+|x|)^{1+k}}
$$

with $k>0$, where $\varepsilon$ is a small parameter.

Remark 2.1. (H2) implies $f(x) \in H^{2}\left(R^{2}\right)$ and $g(x) \in H^{1}\left(R^{2}\right)$. 
We solve the integral equation

$$
\begin{array}{r}
u(x, t)=u_{0}^{-}(x, t)+\frac{1}{2 \pi} \int_{-\infty}^{t} \int_{|x-y| \leq t-s} \frac{F(u(y, s))}{\sqrt{(t-s)^{2}-|x-y|^{2}}} d y d s \\
\text { for } t \in R
\end{array}
$$

in the space $X_{k}, k>2 /(p-1)$, where

$$
X_{k}=\left\{u(x, t): \mathrm{D}_{x}^{\alpha} u(x, t) \in C\left(R^{2} \times R\right) \text { for }|\alpha| \leq 2,\|u\|_{X_{k}}<\infty\right\},
$$

$$
\|u\|_{k}=\left\{\begin{array}{l}
\sup _{\substack{x \in R^{2} \\
t \in R}} \frac{(1+|t|+|x|)^{1 / 2}(1+|| t|-| x||)^{m}}{\ln (2+|| t|-| x||)}|u(x, t)| \quad\left(k>\frac{1}{2}\right), \\
\sup _{\substack{x \in R^{2} \\
t \in R}}(1+|t|+|x|)^{1 / 2}\left(1+\ln \frac{1+|t|+|x|}{1+|| t|-| x||}\right)^{-1}|u(x, t)| \quad\left(k=\frac{1}{2}\right), \\
\sup _{\substack{x \in R^{2} \\
t \in R}}(1+|t|+|x|)^{k}|u(x, t)| \quad\left(0<k<\frac{1}{2}\right),
\end{array}\right.
$$

and

$$
m=\min (1 / 2,(p-3) / 2, k-1 / 2) .
$$

We put

$$
\|u(t)\|_{e}=\left\{\left\|\mathrm{D}_{x} u(t)\right\|_{L^{2}\left(R^{2}\right)}^{2}+\left\|\partial_{t} u(t)\right\|_{L^{2}\left(R^{2}\right)}^{2}\right\}^{1 / 2} .
$$

Our goal is to prove the following

Theorem 2.2. Assume hypotheses (H1) and (H2).

(i) If $k>2 /(p-1)$ and $\varepsilon$ is sufficiently small, depending on $\lambda, p$, and $k$, then there exists a unique $C^{2}$-solution $u(x, t)$ of the integral equation (2.4) such that

$$
\left\|u(t)-u_{0}^{-}(t)\right\|_{e} \leq C /(1+|t|)^{l} \rightarrow 0 \quad(t \rightarrow-\infty),
$$

where $C$ is a constant depending on $\lambda, p, k$, and

$$
l= \begin{cases}m & \left(k>\frac{1}{2}\right), \\ k & \left(0<k \leq \frac{1}{2}\right) .\end{cases}
$$

(ii) Moreover, there exists a unique $C^{2}$-solution $u_{0}^{+}(x, t)$ of $(2.2)$ such that for the solution $u(x, t)$ of (2.4) given by part (i),

$$
\left\|u(t)-u_{0}^{+}(t)\right\|_{e} \leq C /(1+|t|)^{l} \rightarrow 0 \quad(t \rightarrow+\infty) .
$$

Thus, we can define the scattering operator

$$
S:\left(u_{0}^{-}(0), \partial_{t} u_{0}^{-}(0)\right) \rightarrow\left(u_{0}^{+}(0), \partial_{t} u_{0}^{+}(0)\right)
$$

for equation (2.1).

Remark 2.3. The solution $u(x, t)$ of $(2.4)$ given by Theorem 2.2 is a solution of (2.1) (see [2, 7, 8, 16]). 
Corollary 2.4. The well-known energy equality holds:

$$
\frac{1}{2}\|u(t)\|_{e}^{2}+\int_{R^{2}} G(u(t)) d x=\frac{1}{2}\left\|u_{0}^{-}(0)\right\|_{e}^{2}=\frac{1}{2}\left\|u_{0}^{+}(0)\right\|_{e}^{2}, \quad t \in R,
$$

where

$$
G(u)=-\int_{0}^{u} F(s) d s .
$$

The paper is organized as follows. In $\S 3$, we describe the decay estimates for the linear problem which was derived in $[13,22]$. In $\S 4$, we prove the basic estimate for the existence proof, following Kovalyov [11, 12] and Asakura [2]. Finally, Theorem 2.2 and Corollary 2.4 will be proved in $\S 5$.

We denote a constant in the estimate by $C$, which will change from step to step.

\section{Decay estimate}

Consider the Cauchy problem (2.2) and (2.3) for $t \geq 0$ under the hypothesis (H2).

The following lemma is proved in [22] (see also [13]).

Lemma 3.1. Let $f(x), g(x)$ satisfy (H2). Then, the solution $u$ of (2.2) and (2.3) for $t \geq 0$ satisfies

$$
\sum_{|\alpha| \leq 2}\left|\mathrm{D}_{x}^{\alpha} u(x, t)\right| \leq\left\{\begin{array}{lc}
\frac{C_{k} \varepsilon}{\sqrt{(1+t+a)(1+|t-a|)}} & (k>1), \\
\frac{C_{k} \varepsilon \ln (2+|t-a|)}{\sqrt{(1+t+a)(1+|t-a|)}} & (k=1), \\
\frac{C_{k} \varepsilon}{\sqrt{1+t+a}(1+|t-a|)^{k-1 / 2}} & \left(\frac{1}{2}<k<1\right), \\
\frac{C_{k} \varepsilon}{\sqrt{1+t+a}}\left(1+\ln \frac{1+t+a}{1+|t-a|}\right) & \left(k=\frac{1}{2}\right), \\
\frac{C_{k} \varepsilon}{(1+t+a)^{k}} & \left(0<k<\frac{1}{2}\right),
\end{array}\right.
$$

where $a=|x|$, and a constant $C_{k}$ depends only on $k$.

Remark 3.2. The same estimate holds for all $t \in R$ by replacing $t$ by $|t|$.

\section{The basic estimate}

We consider the integral equation

$$
u(x, t)=u_{0}^{-}(x, t)+\frac{1}{2 \pi} \int_{-\infty}^{t} \int_{|x-y| \leq t-s} \frac{F(u(y, s))}{\sqrt{(t-s)^{2}-|x-y|^{2}}} d y d s,
$$

for $t \in R$.

We define

$$
L F(u)(x, t)=\frac{1}{2 \pi} \int_{-\infty}^{t} \int_{|x-y| \leq t-s} \frac{F(u(y, s))}{\sqrt{(t-s)^{2}-|x-y|^{2}}} d y d s .
$$

The following lemma is the basic estimate for the existence proof. 
Lemma 4.1. Assume that $u(x, t) \in C\left(R^{2} \times R\right)$ satisfies

$$
|u(x, t)| \leq \frac{M \ln (2+|| t|-a|)}{\sqrt{1+|t|+a}(1+|| t|-a|)^{m}},
$$

where $a=|x|, m=\min (1 / 2,(p-3) / 2, k-1 / 2)$, and $k>1 / 2$.

Let $k>2 /(p-1)$ and $p>(3+\sqrt{17}) / 2$. Then, there exists a constant $C_{p, k}$ depending only on $p$ and $k$, not on $M$ such that

$$
\left.|L| u\right|^{p}(x, t) \mid \leq \frac{C_{p, k} M^{p} \ln (2+|| t|-a|)}{\sqrt{1+|t|+a}(1+|| t|-a|)^{m}} .
$$

Proof. From (4.1),

$$
\begin{aligned}
L|u|^{p}(x, t) \leq & \frac{1}{2 \pi} \int_{-\infty}^{t} \int_{|x-y| \leq t-s} \\
& \frac{1}{\sqrt{(t-s)^{2}-|x-y|^{2}}} \\
\leq C M^{p} \int_{-\infty}^{t} \int_{|x-y| \leq t-s} & \frac{M^{p}(\ln (2+|| s|-| y||))^{p}}{(1+|s|+|y|)^{p / 2}(1+|| s|-| y||)^{p m}} d y d s \\
& \cdot \frac{1}{\sqrt{(t-s)^{2}-|x-y|^{2}}} \\
& \frac{1}{(1+|s|+|y|)^{p / 2}(1+|| s|-| y||)^{p m-\varepsilon^{\prime}}} d y d s,
\end{aligned}
$$

where $\varepsilon^{\prime}$ is a small positive constant to be determined later and $C$ depends on $\varepsilon^{\prime}$.

Switching the last estimate to polar coordinates, we have

$$
\begin{aligned}
L|u|^{p}(x, t)= & L|u|^{p}(a, \theta, t) \\
= & C M^{p} \int_{t-a}^{t} \int_{a-t+s}^{a+t-s} \frac{r}{(1+|s|+r)^{p / 2}(1+|| s|-r|)^{p m-\varepsilon^{\prime}}} \\
& \cdot \int_{-\varphi}^{\varphi} \frac{1}{\sqrt{(t-s)^{2}-a^{2}-r^{2}+2 a r \cos \psi}} d \psi d r d s \\
& +C M^{p} \int_{-\infty}^{t-a} \int_{t-a-s}^{t+a-s} \frac{r}{(1+|s|+r)^{p / 2}(1+|| s|-r|)^{p m-\varepsilon^{\prime}}} \\
& \cdot \int_{-\varphi}^{\varphi} \frac{1}{\sqrt{(t-s)^{2}-a^{2}-r^{2}+2 a r \cos \psi}} d \psi d r d s \\
& +C M^{p} \int_{-\infty}^{t-a} \int_{0}^{t-a-s} \frac{r}{(1+|s|+r)^{p / 2}(1+|| s|-r|)^{p m-\varepsilon^{\prime}}} \\
& \cdot \int_{-\pi}^{\pi} \frac{1}{\sqrt{(t-s)^{2}-a^{2}-r^{2}+2 a r \cos \psi}} d \psi d r d s,
\end{aligned}
$$

where $\varphi=\arccos \left(\left(a^{2}+r^{2}-(t-s)^{2}\right) / 2 a r\right), x=(a \cos \theta, a \sin \theta), \quad y=$ $(r \cos (\theta+\psi), r \sin (\theta+\psi))$ (see Kovalyov [11, 12]).

We define

$$
K(t, a, r)= \begin{cases}\int_{-\varphi}^{\varphi} \frac{1}{\sqrt{t^{2}-a^{2}-r^{2}+2 a r \cos \psi}} d \psi & \text { if }\left|\frac{a^{2}+r^{2}-t^{2}}{2 a r}\right| \leq 1, \\ \int_{-\pi}^{\pi} \frac{1}{\sqrt{t^{2}-a^{2}-r^{2}+2 a r \cos \psi}} d \psi & \text { if }\left|\frac{a^{2}+r^{2}-t^{2}}{2 a r}\right| \geq 1,\end{cases}
$$


where

$$
\varphi=\arccos \frac{a^{2}+r^{2}-t^{2}}{2 a r} .
$$

We use the following lemma which is proved in [11, 12] (see also [22]).

Lemma 4.2. (i) If $t \geq a+r$ and $\left|\left(a^{2}+r^{2}-t^{2}\right) / 2 a r\right| \geq 1$, then

$$
\begin{aligned}
K(t, a, r) & \leq \frac{C \ln \left[2+a r /\left(t^{2}-(a+r)^{2}\right)\right]}{\sqrt{t^{2}-a^{2}-r^{2}}} \\
& \leq \frac{C}{\sqrt{t^{2}-(a+r)^{2}}} .
\end{aligned}
$$

(ii) If $t \leq a+r$ and $\left|\left(a^{2}+r^{2}-t^{2}\right) / 2 a r\right| \leq 1$, then

$$
\begin{aligned}
K(t, a, r) & \leq \frac{C}{\sqrt{a r}} \ln \left[2+\frac{a r \chi(t-a)}{(a+r)^{2}-t^{2}}\right] \\
& \leq \frac{C}{\sqrt{(a+r)^{2}-t^{2}}},
\end{aligned}
$$

where $\chi$ is the characteristic function of positive numbers.

From (4.5) and (4.6), we have

$$
\begin{aligned}
L|u|^{p}(x, t) \leq & C M^{p} \int_{D^{\prime}} \frac{K(t-s, a, r) r}{(1+r+|s|)^{p / 2}(1+|r-| s||)^{p m-\varepsilon^{\prime}}} d r d s \\
& +C M^{p} \int_{D^{\prime \prime}} \frac{K(t-s, a, r) r}{(1+r+|s|)^{p / 2}(1+|r-| s||)^{p m-\varepsilon^{\prime}}} d r d s \\
\equiv & \mathrm{I}+\mathrm{II},
\end{aligned}
$$

where the domains $D^{\prime}$ and $D^{\prime \prime}$ denote the sets

$$
\begin{aligned}
D^{\prime} & =\{(s, r):|s+a-t| \leq r \leq-s+t+a,-\infty<s \leq t\}, \\
D^{\prime \prime} & =\{(s, r): 0 \leq r \leq-s+t-a,-\infty<s \leq t-a\}
\end{aligned}
$$

(see $[11,12])$.

By $(4.8 \mathrm{a})$ of Lemma 4.2,

Since

$$
\mathrm{I} \leq \frac{C M^{p}}{\sqrt{a}} \int_{D^{\prime}} \frac{\sqrt{r} \ln \left[2+\operatorname{ar} \chi(t-s-a) /\left((a+r)^{2}-(t-s)^{2}\right)\right]}{(1+r+|s|)^{p / 2}(1+|r-| s||)^{p m-\varepsilon^{\prime}}} d r d s .
$$

$$
\begin{gathered}
2+\frac{a r}{(a+r+t-s)(a+r-t+s)} \leq(2+r)\left(1+\frac{1}{a+r-t+s}\right), \\
\ln \left(1+\frac{1}{a+r-t+s}\right) \leq \frac{1}{\sqrt{a+r-t+s}}
\end{gathered}
$$

we obtain

$$
\mathrm{I} \leq\left\{\begin{array}{r}
\frac{C M^{p}}{\sqrt{a}} \int_{D^{\prime}} \frac{\ln (2+r)+1 / \sqrt{a+r-t+s}}{(1+r+|s|)^{(p-1) / 2}(1+|r-| s||)^{p m-\varepsilon^{\prime}}} d r d s \\
\frac{C M^{p}}{\sqrt{a}} \int_{D^{\prime}} \frac{1}{(t-s-a \geq 0),} d r d s \\
(t-s-a \leq 0),
\end{array}\right.
$$


or by $(4.8 b)$,

(4.11)

$\mathrm{I} \leq \int_{D^{\prime}} \frac{C M^{p}}{\sqrt{(a+r+t-s)(a+r-t+s)}(1+r+|s|)^{p / 2-1}(1+|r-| s||)^{p m-\varepsilon^{\prime}}} d r d s$.

Using (4.7a) of Lemma 4.2,

$$
\mathrm{II} \leq C M^{p} \int_{D^{\prime \prime}} \frac{r \ln \left[2+a r /\left((t-s)^{2}-(a+r)^{2}\right)\right]}{\sqrt{(t-s)^{2}-a^{2}-r^{2}}(1+r+|s|)^{p / 2}(1+|r-| s||)^{p m-\varepsilon^{\prime}}} d r d s .
$$

Note that

$$
\begin{aligned}
2+\frac{a r}{(t-s+a+r)(t-s-a-r)} & \leq(2+r)\left(1+\frac{1}{t-s-a-r}\right), \\
\ln \left(1+\frac{1}{t-s-a-r}\right) & \leq \frac{1}{\sqrt{t-s-a-r}}, \\
\sqrt{(t-s)^{2}-a^{2}-r^{2}} & \geq \sqrt{2 a(t-s-a)},
\end{aligned}
$$

since $r \leq t-s-a$. Then, we obtain

$$
\mathrm{II} \leq \frac{C M^{p}}{\sqrt{a}} \int_{D^{\prime \prime}} \frac{\ln (2+r)+1 / \sqrt{t-s-a-r}}{(1+r+|s|)^{(p-1) / 2}(1+|r-| s||)^{p m-\varepsilon^{\prime}}} d r d s,
$$

or by $(4.7 b)$,

$$
\mathrm{II} \leq \int_{D^{\prime \prime}} \frac{C M^{p}}{\sqrt{(t-s+a+r)(t-s-a-r)}(1+r+|s|)^{p / 2-1}(1+|r-| s||)^{p m-\varepsilon^{\prime}}} d r d s .
$$

Now, we shall estimate I and II by dividing into four cases.

Case $1 .|t| \geq a$ and $t \geq 0$.

Introducing the following change of variables

$$
\alpha=r+s, \quad \beta=r-s,
$$

the domains of integration $D^{\prime}$ and $D^{\prime \prime}$ go to $D_{1}^{\prime} \cup D_{2}^{\prime}$ and $D_{1}^{\prime \prime} \cup D_{2}^{\prime \prime} \cup D_{3}^{\prime \prime} \cup D_{4}^{\prime \prime}$ respectively, where $D_{i}^{\prime}(i=1,2)$ and $D_{j}^{\prime \prime}(j=1,2,3,4)$ denote the sets

$$
\begin{aligned}
& D_{1}^{\prime}=\{(\alpha, \beta): t-a \leq \alpha \leq t+a, a-t \leq \beta \leq \alpha\}, \\
& D_{2}^{\prime}=\{(\alpha, \beta): t-a \leq \alpha \leq t+a, \alpha \leq \beta\}, \\
& D_{1}^{\prime \prime}=\{(\alpha, \beta): 0 \leq \alpha \leq t-a,-\alpha \leq \beta \leq \alpha\}, \\
& D_{2}^{\prime \prime}=\{(\alpha, \beta): 0 \leq \beta \leq t-a,-\beta \leq \alpha \leq \beta\}, \\
& D_{3}^{\prime \prime}=\{(\alpha, \beta): a-t \leq \alpha \leq t-a, t-a \leq \beta\}, \\
& D_{4}^{\prime \prime}=\{(\alpha, \beta): \alpha \leq a-t,-\alpha \leq \beta\} .
\end{aligned}
$$

These sets are pictured in Figure 1.

Note that $D_{1}^{\prime}, D_{1}^{\prime \prime}$ corresponds to $D^{\prime} \cap\{(s, r): s \geq 0\}, D^{\prime \prime} \cap\{(s, r): s \geq 0\}$ and $D_{2}^{\prime}, D_{2}^{\prime \prime} \cup D_{3}^{\prime \prime} \cup D_{4}^{\prime \prime}$ corresponds to $D^{\prime} \cap\{(s, r): s \leq 0\}, D^{\prime \prime} \cap\{(s, r): s \leq 0\}$ respectively.

We denote the integrals in (4.9)-(4.13) over the domains $D_{i}^{\prime}(i=1,2), D_{j}^{\prime \prime}$ $(j=1,2,3,4)$ by $\mathbf{I}_{i}, \mathrm{II}_{j}$ respectively. 


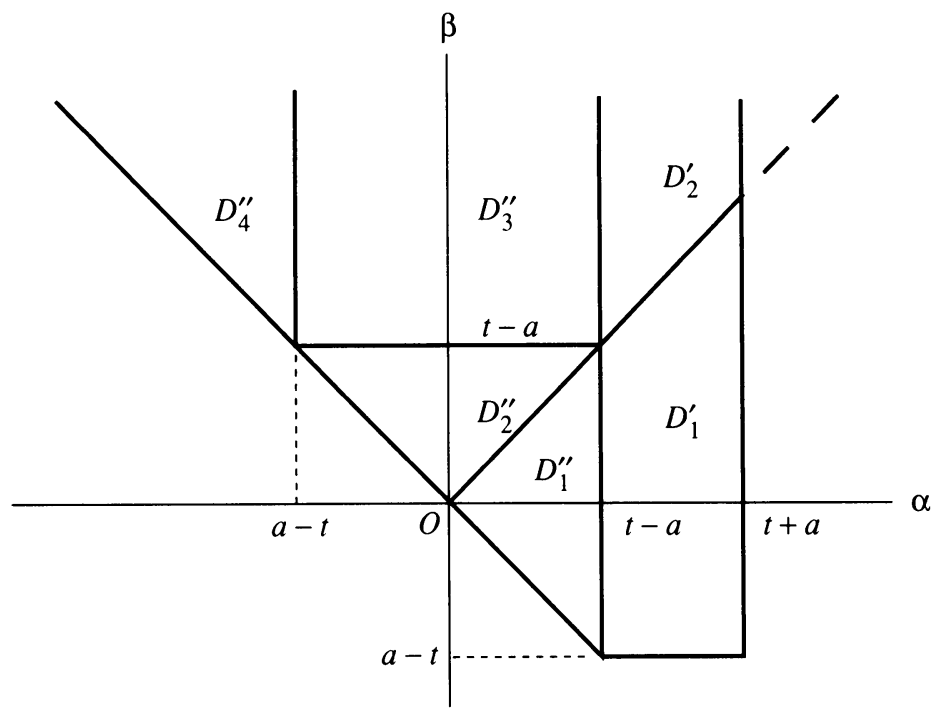

FIGURE 1

$I_{1}$. We begin by estimating $I_{1}$. From (4.11), changing variables by (4.14), we have

(4.15)

$$
\begin{aligned}
\mathrm{I}_{1} \leq & C M^{p} \int_{t-a}^{t+a} \frac{1}{\sqrt{a+\alpha-t}(1+\alpha)^{p / 2-1}} \int_{a-t}^{\alpha} \frac{1}{\sqrt{a+t+\beta}(1+|\beta|)^{p m-\varepsilon^{\prime}}} d \beta d \alpha \\
= & C M^{p} \int_{t-a}^{t+a} \frac{1}{\sqrt{a+\alpha-t}(1+\alpha)^{p / 2-1}} \int_{0}^{\alpha} \frac{1}{\sqrt{a+t+\beta}(1+\beta)^{p m-\varepsilon^{\prime}}} d \beta d \alpha \\
& +C M^{p} \int_{t-a}^{t+a} \frac{1}{\sqrt{a+\alpha-t}(1+\alpha)^{p / 2-1}} \int_{0}^{t-a} \frac{1}{\sqrt{a+t-\beta}(1+\beta)^{p m-\varepsilon^{\prime}}} d \beta d \alpha .
\end{aligned}
$$

We use the following lemma.

\section{Lemma 4.3.}

$$
\int_{0}^{\alpha} \frac{1}{\sqrt{a+t \pm \beta}(1+\beta)^{k}} d \beta \leq \begin{cases}\frac{C}{\sqrt{t+a}} & (k>1), \\ \frac{C \ln (1+\alpha)}{\sqrt{t+a}} & (k=1), \\ \frac{C(1+\alpha)^{1-k}}{\sqrt{t+a}} & (0<k<1)\end{cases}
$$

for $0 \leq \alpha \leq t+a$, where $C$ is a constant depending only on $k$.

Proof. The proof essentially repeats the proof of Lemma 4.3 in [22].

Now, consider $p m-\varepsilon^{\prime}$.

$p \geq 4$, if $m=\frac{1}{2}$. Hence, we can choose $\varepsilon^{\prime}$ such that

$$
p / 2-\varepsilon^{\prime}>3 / 2 \text {. }
$$

If $m=(p-3) / 2$, since $p>(3+\sqrt{17}) / 2$,

$$
p(p-3) / 2-\varepsilon^{\prime}>1 \text {. }
$$


If $m=k-\frac{1}{2}$ and $p\left(k-\frac{1}{2}\right)>1$,

$$
p\left(k-\frac{1}{2}\right)-\varepsilon^{\prime}>1 \text {. }
$$

Hence, for these three cases, applying Lemma 4.3 to (4.15) we have

$$
\mathrm{I}_{1} \leq \frac{C M^{p}}{\sqrt{t+a}} \int_{t-a}^{t+a} \frac{1}{\sqrt{a+\alpha-t}(1+\alpha)^{p / 2-1}} d \alpha .
$$

On the other hand, if $m=k-\frac{1}{2}$ and $p\left(k-\frac{1}{2}\right) \leq 1$, as above

$$
\mathrm{I}_{1} \leq \frac{C M^{p}}{\sqrt{t+a}} \int_{t-a}^{t+a} \frac{1}{\sqrt{a+\alpha-t}(1+\alpha)^{p k-2-\varepsilon^{\prime}}} d \alpha .
$$

The following lemma is proved in [22].

\section{Lemma 4.4.}

$$
\begin{aligned}
& \frac{1}{\sqrt{a}} \int_{|t-a|}^{t+a} \frac{1}{\sqrt{a+\alpha-t}(1+\alpha)^{k}} d \alpha \\
& \quad \leq \begin{cases}\frac{C}{\sqrt{1+t+a}(1+|t-a|)^{k-1 / 2}} & \left(k>\frac{1}{2}\right), \\
\frac{C}{\sqrt{1+t+a}}\left(1+\ln \frac{1+t+a}{1+|t-a|}\right) & \left(k=\frac{1}{2}\right), \\
\frac{C}{(1+t+a)^{k}} & \left(0<k<\frac{1}{2}\right)\end{cases}
\end{aligned}
$$

for all $t, a \geq 0$.

Combining (4.22) with (4.20), we obtain

$$
\mathrm{I}_{1} \leq \frac{C M^{p}}{\sqrt{1+t+a}(1+t-a)^{(p-3) / 2}} \leq \frac{C M^{p}}{\sqrt{1+t+a}(1+t-a)^{m}} .
$$

For (4.21), as above

$$
\mathrm{I}_{1} \leq \frac{C M^{p}}{\sqrt{1+t+a}(1+t-a)^{p k-5 / 2-\varepsilon^{\prime}}} \leq \frac{C M^{p}}{\sqrt{1+t+a}(1+t-a)^{k-1 / 2}} .
$$

In the last inequality we have used that

$$
p k-k-\varepsilon^{\prime}>2 \text {, }
$$

since $k>2 /(p-1)$.

$I_{2}$. Next, we shall estimate $I_{2}$. From (4.9) and changing variables by (4.14), we have

$$
\begin{aligned}
\mathrm{I}_{2} \leq & \frac{C M^{p}}{\sqrt{a}} \int_{t-a}^{t+a} \frac{1}{(1+\alpha)^{p m-\varepsilon^{\prime}}} \int_{\alpha}^{\infty} \frac{\ln (2+\beta)}{(1+\beta)^{(p-1) / 2}} d \beta d \alpha \\
& +\frac{C M^{p}}{\sqrt{a}} \int_{t-a}^{t+a} \frac{1}{\sqrt{a+\alpha-t}(1+\alpha)^{p m-\varepsilon^{\prime}}} \int_{\alpha}^{\infty} \frac{1}{(1+\beta)^{(p-1) / 2}} d \beta d \alpha \\
\leq & \frac{C M^{p}}{\sqrt{a}} \int_{t-a}^{t+a} \frac{1}{(1+\alpha)^{p m+(p-3) / 2-\varepsilon^{\prime}-\varepsilon^{\prime \prime}}} d \alpha \\
& +\frac{C M^{p}}{\sqrt{a}} \int_{t-a}^{t+a} \frac{1}{\sqrt{a+\alpha-t}(1+\alpha)^{p m+(p-3) / 2-\varepsilon^{\prime}}} d \alpha
\end{aligned}
$$


where $\varepsilon^{\prime \prime}$ is a small positive constant to be determined later and $C$ depends on $\varepsilon^{\prime \prime}$.

To continue, we need the following lemma which is proved in [22].

\section{Lemma 4.5.}

for all $t, a \geq 0$.

$$
\begin{aligned}
& \frac{1}{\sqrt{a}} \int_{|t-a|}^{t+a} \frac{1}{(1+\alpha)^{k+1 / 2}} d \alpha \\
& \quad \leq \begin{cases}\frac{C}{\sqrt{1+t+a}(1+|t-a|)^{k-1 / 2}} & \left(k>\frac{1}{2}\right), \\
\frac{C}{\sqrt{1+t+a}}\left(1+\ln \frac{1+t+a}{1+|t-a|}\right) & \left(k=\frac{1}{2}\right), \\
\frac{C}{(1+t+a)^{k}} & \left(0<k<\frac{1}{2}\right)\end{cases}
\end{aligned}
$$

$p \geq 4$, if $m=\frac{1}{2}$. Hence, we can choose $\varepsilon^{\prime}, \varepsilon^{\prime \prime}$ such that

$$
p-3 / 2-\varepsilon^{\prime}-\varepsilon^{\prime \prime}>3 / 2 \text {. }
$$

Thus, applying Lemmas 4.4 and 4.5 to (4.27), we obtain

$$
\mathrm{I}_{2} \leq \frac{C M^{p}}{\sqrt{(1+t+a)(1+t-a)}} .
$$

If $m=(p-3) / 2$, since $p>(3+\sqrt{17}) / 2$,

$$
p(p-3) / 2+(p-3) / 2-\varepsilon^{\prime}-\varepsilon^{\prime \prime}>(p-1) / 2 .
$$

Thus, as above

$$
\mathrm{I}_{2} \leq \frac{C M^{p}}{\sqrt{1+t+a}(1+t-a)^{(p-3) / 2}} .
$$

If $m=k-\frac{1}{2}$, since $k>2 /(p-1)$,

$$
p k-3 / 2-\varepsilon^{\prime}-\varepsilon^{\prime \prime}>k+1 / 2 \text {. }
$$

Thus, as above

$$
\mathrm{I}_{2} \leq \frac{C M^{p}}{\sqrt{1+t+a}(1+t-a)^{k-1 / 2}} .
$$

$\mathrm{II}_{1}$. To estimate $\mathrm{II}_{1}$, we make the change of variables by (4.14). Then, from (4.13),

$$
\begin{aligned}
\mathrm{II}_{1} \leq & C M^{p} \int_{0}^{t-a} \frac{1}{\sqrt{t-a-\alpha}(1+\alpha)^{p / 2-1}} \int_{-\alpha}^{\alpha} \frac{1}{\sqrt{t+a+\beta}(1+|\beta|)^{p m-\varepsilon^{\prime}}} d \beta d \alpha \\
= & C M^{p} \int_{0}^{t-a} \frac{1}{\sqrt{t-a-\alpha}(1+\alpha)^{p / 2-1}} \int_{0}^{\alpha} \frac{1}{\sqrt{t+a+\beta}(1+\beta)^{p m-\varepsilon^{\prime}}} d \beta d \alpha \\
& +C M^{p} \int_{0}^{t-a} \frac{1}{\sqrt{t-a-\alpha}(1+\alpha)^{p / 2-1}} \int_{0}^{\alpha} \frac{1}{\sqrt{t+a-\beta}(1+\beta)^{p m-\varepsilon^{\prime}}} d \beta d \alpha .
\end{aligned}
$$

We subdivide into two cases. 
(a) $m=\frac{1}{2},(p-3) / 2$, or $m=k-\frac{1}{2}$ and $p\left(k-\frac{1}{2}\right)>1$. Using Lemma 4.3 and by (4.17), (4.18), and (4.19), we have

$$
\mathrm{II}_{1} \leq \frac{C M^{p}}{\sqrt{t+a}} \int_{0}^{t-a} \frac{1}{\sqrt{t-a-\alpha}(1+\alpha)^{p / 2-1}} d \alpha .
$$

We make use of the following lemma, which will be also used repeatedly in this section.

\section{Lemma 4.6.}

$$
\begin{aligned}
& \frac{1}{\sqrt{t+a}} \int_{0}^{\beta} \frac{1}{\sqrt{t-a \pm \alpha}(1+\alpha)^{k}} d \alpha \\
& \quad \leq \begin{cases}\frac{C}{\sqrt{(1+t+a)(1+t-a)}} & (k>1), \\
\frac{C \ln (2+\beta)}{\sqrt{(1+t+a)(1+t-a)}} & (k=1), \\
\frac{C(1+\beta)^{1-k}}{\sqrt{(1+t+a)(1+t-a)}} & (0<k<1)\end{cases}
\end{aligned}
$$

for $0 \leq \beta \leq t-a$, all $t \geq a \geq 0$.

Proof. The proof is almost the same as that of Lemma 3.7 in [22], where we estimated

$$
\frac{1}{\sqrt{t+a}} \int_{0}^{t-a} \frac{1}{\sqrt{t-a-\alpha}(1+\alpha)^{k}} d \alpha .
$$

By Lemma 4.6, we obtain

$$
\mathrm{II}_{1} \leq \begin{cases}\frac{C M^{p}}{\sqrt{(1+t+a)(1+t-a)}} & (p>4), \\ \frac{C M^{p} \ln (2+t-a)}{\sqrt{(1+t+a)(1+t-a)}} & (p=4), \\ \frac{C M^{p}}{\sqrt{1+t+a}(1+t-a)^{(p-3) / 2}} & \left(\frac{3+\sqrt{17}}{2}<p<4\right) .\end{cases}
$$

Thus,

$$
\mathrm{II}_{1} \leq \frac{C M^{p} \ln (2+t-a)}{\sqrt{1+t+a}(1+t-a)^{m}} .
$$

(b) $m=k-\frac{1}{2}$ and $p\left(k-\frac{1}{2}\right) \leq 1$. Applying Lemmas 4.3 and 4.6 to (4.31), by (4.25) and $k \leq 1 / 2+1 / p<1$, we obtain

$$
\begin{aligned}
\mathrm{II}_{1} & \leq \frac{C M^{p}}{\sqrt{t+a}} \int_{0}^{t-a} \frac{1}{\sqrt{t-a-\alpha}(1+\alpha)^{p k-2-\varepsilon^{\prime}}} d \alpha \\
& \leq \frac{C M^{p}}{\sqrt{1+t+a}(1+t-a)^{k-1 / 2}} .
\end{aligned}
$$


$\mathrm{II}_{2}$. From (4.13),

(4.36)

$$
\begin{aligned}
\mathrm{II}_{2} \leq & C M^{p} \int_{0}^{t-a} \frac{1}{\sqrt{t+a+\beta}(1+\beta)^{p / 2-1}} \int_{-\beta}^{\beta} \frac{1}{\sqrt{t-a-\alpha}(1+|\alpha|)^{p m-\varepsilon^{\prime}}} d \alpha d \beta \\
\leq & \frac{C M^{p}}{\sqrt{t+a}} \int_{0}^{t-a} \frac{1}{(1+\beta)^{p / 2-1}} \int_{0}^{\beta} \frac{1}{\sqrt{t-a-\alpha}(1+\alpha)^{p m-\varepsilon^{\prime}}} d \alpha d \beta \\
& +\frac{C M^{p}}{\sqrt{t+a}} \int_{0}^{t-a} \frac{1}{(1+\beta)^{p / 2-1}} \int_{0}^{\beta} \frac{1}{\sqrt{t-a+\alpha}(1+\alpha)^{p m-\varepsilon^{\prime}}} d \alpha d \beta .
\end{aligned}
$$

(a) $m=\frac{1}{2},(p-3) / 2$, or $m=k-\frac{1}{2}$ and $p\left(k-\frac{1}{2}\right)>1$. Using Lemma 4.6, (4.17), (4.18), and (4.19),

$$
\begin{aligned}
\mathrm{II}_{2} & \leq \frac{C M^{p}}{\sqrt{(1+t+a)(1+t-a)}} \int_{0}^{t-a} \frac{1}{(1+\beta)^{p / 2-1}} d \beta \\
& \leq\left\{\begin{array}{lc}
\frac{C M^{p}}{\sqrt{(1+t+a)(1+t-a)}} & (p>4), \\
\frac{C M^{p} \ln (2+t-a)}{\sqrt{(1+t+a)(1+t-a)}} & (p=4), \\
\frac{C M^{p}}{\sqrt{1+t+a}(1+t-a)^{(p-3) / 2}} & \left(\frac{3+\sqrt{17}}{2}<p<4\right) .
\end{array}\right.
\end{aligned}
$$

Thus,

$$
\mathrm{II}_{2} \leq \frac{C M^{p} \ln (2+t-a)}{\sqrt{1+t+a}(1+t-a)^{m}}
$$

(b) $m=k-\frac{1}{2}$ and $p\left(k-\frac{1}{2}\right) \leq 1$. We use Lemma 4.6 to obtain by (4.25) and $k \leq 1 / 2+1 / p<1$,

$$
\begin{aligned}
\mathrm{II}_{2} & \leq \frac{C M^{p}}{\sqrt{(1+t+a)(1+t-a)}} \int_{0}^{t-a} \frac{1}{(1+\beta)^{p k-2-\varepsilon^{\prime}}} d \beta \\
& \leq \frac{C M^{p}}{\sqrt{1+t+a}(1+t-a)^{k-1 / 2}} .
\end{aligned}
$$

$\mathrm{II}_{3}$. To estimate $\mathrm{II}_{3}$, we distinguish two cases.

(i) $t \geq 2 a$, or $2 a \geq t \geq a$ and $a \leq 1$. From (4.13),

$\mathrm{II}_{3} \leq C M^{p} \int_{a-t}^{t-a} \frac{1}{\sqrt{t-a-\alpha}(1+|\alpha|)^{p m-\varepsilon^{\prime}}} d \alpha \int_{t-a}^{\infty} \frac{1}{\sqrt{t+a+\beta}(1+\beta)^{p / 2-1}} d \beta$.

We can easily derive

$$
\int_{a}^{\infty} \frac{1}{\sqrt{b+\alpha}(1+\alpha)^{k}} d \alpha \leq \frac{C}{(1+a)^{k-1 / 2}} \text { for } k>\frac{1}{2} \text { and } a, b \geq 0 .
$$

Hence,

$$
\mathrm{II}_{3} \leq \frac{C M^{p}}{(1+t-a)^{(p-3) / 2}} \int_{0}^{t-a}\left(\frac{1}{\sqrt{t-a-\alpha}}+\frac{1}{\sqrt{t-a+\alpha}}\right) \frac{1}{(1+\alpha)^{p m-\varepsilon^{\prime}}} d \alpha .
$$


(a) $m=\frac{1}{2},(p-3) / 2$, or $m=k-\frac{1}{2}$ and $p\left(k-\frac{1}{2}\right)>1$. By Lemma 4.6,

$$
\mathrm{II}_{3} \leq \frac{C M^{p}}{(1+t-a)^{(p-2) / 2}} .
$$

$t-a \geq(t+a) / 3$, if $t \geq 2 a$. Thus,

$$
\mathrm{II}_{3} \leq \frac{C M^{p}}{\sqrt{1+t+a}(1+t-a)^{(p-3) / 2}} \leq \frac{C M^{p}}{\sqrt{1+t+a}(1+t-a)^{m}} .
$$

On the other hand, since the right-hand side of (4.41) is bounded, also for $2 a \geq t \geq a$ and $a \leq 1,(4.42)$ holds.

(b) $m=k-\frac{1}{2}$ and $p\left(k-\frac{1}{2}\right) \leq 1$. By Lemma 4.6, as above

$$
\mathrm{II}_{3} \leq \frac{C M^{p}}{(1+t-a)^{p k-2-\varepsilon^{\prime}}} \leq \frac{C M^{p}}{\sqrt{1+t+a}(1+t-a)^{k-1 / 2}} .
$$

(ii) $2 a \geq t \geq a$ and $a \geq 1$. From (4.12),

$$
\begin{aligned}
\mathrm{II}_{3} \leq & \frac{C M^{p}}{\sqrt{a}} \int_{a-t}^{t-a} \frac{1}{(1+|\alpha|)^{p m-\varepsilon^{\prime}}} d \alpha \int_{t-a}^{\infty} \frac{\ln (2+\beta)}{(1+\beta)^{(p-1) / 2}} d \beta \\
& +\frac{C M^{p}}{\sqrt{a}} \int_{a-t}^{t-a} \frac{1}{\sqrt{t-a-\alpha}(1+|\alpha|)^{p m-\varepsilon^{\prime}}} d \alpha \int_{t-a}^{\infty} \frac{1}{(1+\beta)^{(p-1) / 2}} d \beta \\
\leq & \frac{C M^{p} \ln (2+t-a)}{\sqrt{1+t+a}(1+t-a)^{(p-3) / 2}} \int_{0}^{t-a} \frac{1}{(1+\alpha)^{p m-\varepsilon^{\prime}}} d \alpha \\
& +\frac{C M^{p}}{\sqrt{1+t+a}(1+t-a)^{(p-3) / 2}} \\
& \times \int_{0}^{t-a}\left(\frac{1}{\sqrt{t-a-\alpha}}+\frac{1}{\sqrt{t-a+\alpha}}\right) \frac{1}{(1+\alpha)^{p m-\varepsilon^{\prime}}} d \alpha .
\end{aligned}
$$

(a) $m=\frac{1}{2},(p-3) / 2$, or $m=k-\frac{1}{2}$ and $p\left(k-\frac{1}{2}\right)>1$. Using Lemma 4.6,

$$
\begin{aligned}
\mathrm{II}_{3} & \leq \frac{C M^{p} \ln (2+t-a)}{\sqrt{1+t+a}(1+t-a)^{(p-3) / 2}}+\frac{C M^{p}}{\sqrt{1+t+a}(1+t-a)^{(p-2) / 2}} \\
& \leq \frac{C M^{p} \ln (2+t-a)}{\sqrt{1+t+a}(1+t-a)^{m}} .
\end{aligned}
$$

(b) $m=k-\frac{1}{2}$ and $p\left(k-\frac{1}{2}\right) \leq 1$. As above

$$
\begin{aligned}
\mathrm{II}_{3} & \leq \frac{C M^{p} \ln (2+t-a)}{\sqrt{1+t+a}(1+t-a)^{p k-5 / 2-\varepsilon^{\prime}}}+\frac{C M^{p}}{\sqrt{1+t+a}(1+t-a)^{p k-2-\varepsilon^{\prime}}} \\
& \leq \frac{C M^{p} \ln (2+t-a)}{\sqrt{1+t+a}(1+t-a)^{k-1 / 2}} .
\end{aligned}
$$

$\mathrm{II}_{4}$. As before, we divide into two cases.

(i) $t \geq 2 a$, or $2 a \geq t \geq a$ and $a \leq 1$. By (4.13) and (4.39),

$$
\begin{aligned}
\mathrm{II}_{4} & \leq C M^{p} \int_{-\infty}^{a-t} \frac{1}{\sqrt{t-a-\alpha}(1+|\alpha|)^{p m-\varepsilon^{\prime}}} \int_{-\alpha}^{\infty} \frac{1}{\sqrt{t+a+\beta}(1+\beta)^{p / 2-1}} d \beta d \alpha \\
& \leq C M^{p} \int_{t-a}^{\infty} \frac{1}{\sqrt{t-a+\alpha}(1+\alpha)^{p m+(p-3) / 2-\varepsilon^{\prime}}} d \alpha .
\end{aligned}
$$


We can choose $\varepsilon^{\prime}$ such that $p m+(p-3) / 2-\varepsilon^{\prime}>m+1$. Hence, also using (4.39), we have

$$
\mathrm{II}_{4} \leq \frac{C M^{p}}{(1+t-a)^{m+1 / 2}}
$$

$t-a \geq(t+a) / 3$, if $t \geq 2 a$. Thus, we obtain

$$
\mathrm{II}_{4} \leq \frac{C M^{p}}{\sqrt{1+t+a}(1+t-a)^{m}}
$$

Also for $2 a \geq t \geq a$ and $a \leq 1,(4.48)$ holds.

(ii) $2 a \geq t \geq a$ and $a \geq 1$. From (4.12),

$$
\begin{aligned}
\mathrm{II}_{4} \leq & \frac{C M^{p}}{\sqrt{a}} \int_{-\infty}^{a-t} \frac{1}{(1+|\alpha|)^{p m-\varepsilon^{\prime}}} \int_{-\alpha}^{\infty} \frac{\ln (2+\beta)}{(1+\beta)^{(p-1) / 2}} d \beta d \alpha \\
& +\frac{C M^{p}}{\sqrt{a}} \int_{-\infty}^{a-t} \frac{1}{\sqrt{t-a-\alpha}(1+|\alpha|)^{p m-\varepsilon^{\prime}}} \int_{-\alpha}^{\infty} \frac{1}{(1+\beta)^{(p-1) / 2}} d \beta d \alpha \\
\leq & \frac{C M^{p}}{\sqrt{1+t+a}} \int_{t-a}^{\infty} \frac{1}{(1+\alpha)^{p m+(p-3) / 2-\varepsilon^{\prime}-\varepsilon^{\prime \prime}}} d \alpha \\
& +\frac{C M^{p}}{\sqrt{1+t+a}} \int_{t-a}^{\infty} \frac{1}{\sqrt{t-a+\alpha}(1+\alpha)^{p m+(p-3) / 2-\varepsilon^{\prime}}} d \alpha .
\end{aligned}
$$

We can choose $\varepsilon^{\prime}, \varepsilon^{\prime \prime}$ such that $p m+(p-3) / 2-\varepsilon^{\prime}-\varepsilon^{\prime \prime}>m+1$. Hence, using (4.39), we obtain

$$
\begin{aligned}
\mathrm{II}_{4} & \leq \frac{C M^{p}}{\sqrt{1+t+a}(1+t-a)^{m}}+\frac{C M^{p}}{\sqrt{1+t+a}(1+t-a)^{m+1 / 2}} \\
& \leq \frac{C M^{p}}{\sqrt{1+t+a}(1+t-a)^{m}} .
\end{aligned}
$$

Case 2. $a \geq|t|$ and $t \geq 0$. As in Case 1, changing variables by (4.14), the domains of integration $D^{\prime}$ and $D^{\prime \prime}$ go correspondingly to $D_{1}^{\prime} \cup D_{2}^{\prime} \cup D_{3}^{\prime}$ and $D_{\alpha, \beta}^{\prime \prime}$, where $D_{1}^{\prime}, D_{2}^{\prime}, D_{3}^{\prime}, D_{\alpha, \beta}^{\prime \prime}$ denote the sets

$$
\begin{aligned}
& D_{1}^{\prime}=\{(\alpha, \beta): a-t \leq \alpha \leq t+a, a-t \leq \beta \leq \alpha\} \\
& D_{2}^{\prime}=\{(\alpha, \beta): a-t \leq \alpha \leq t+a, \quad \alpha \leq \beta\} \\
& D_{3}^{\prime}=\{(\alpha, \beta): t-a \leq \alpha \leq a-t, a-t \leq \beta\} \\
& D_{\alpha, \beta}^{\prime \prime}=\{(\alpha, \beta): \alpha \leq t-a,-\alpha \leq \beta\}
\end{aligned}
$$

These sets are shown in Figure 2.

We denote the integrals in (4.9)-(4.11) over the domains $D_{1}^{\prime}, D_{2}^{\prime}, D_{3}^{\prime}$ by $\mathrm{I}_{1}, \mathrm{I}_{2}, \mathrm{I}_{3}$ respectively.

$I_{1}$. From (4.10),

$$
\mathrm{I}_{1} \leq \frac{C M^{p}}{\sqrt{a}} \int_{a-t}^{t+a} \frac{1}{(1+\alpha)^{(p-1) / 2}} \int_{a-t}^{\alpha} \frac{1}{(1+\beta)^{p m-\varepsilon^{\prime}}} d \beta d \alpha
$$




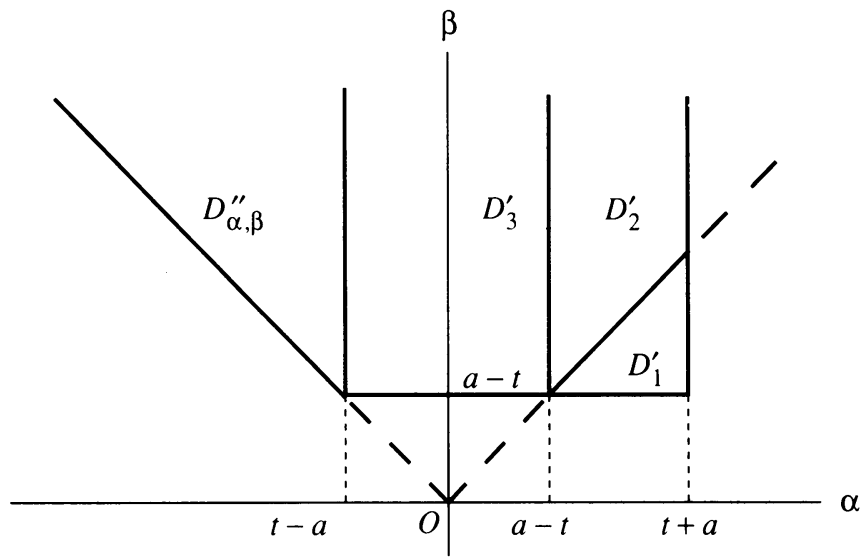

FIGURE 2

(a) $m=\frac{1}{2},(p-3) / 2$, or $m=k-\frac{1}{2}$ and $p\left(k-\frac{1}{2}\right)>1$. By Lemma 4.5,

$$
\begin{aligned}
\mathrm{I}_{1} & \leq \frac{C M^{p}}{\sqrt{a}} \int_{a-t}^{t+a} \frac{1}{(1+\alpha)^{(p-1) / 2}} d \alpha \\
& \leq \frac{C M^{p}}{\sqrt{1+t+a}(1+a-t)^{(p-3) / 2}} \\
& \leq \frac{C M^{p}}{\sqrt{1+t+a}(1+a-t)^{m}} .
\end{aligned}
$$

(b) $m=k-\frac{1}{2}$ and $p\left(k-\frac{1}{2}\right) \leq 1$.

$$
\mathrm{I}_{1} \leq \frac{C M^{p}}{\sqrt{a}} \int_{a-t}^{t+a} \frac{1}{(1+\alpha)^{p k-3 / 2-\varepsilon^{\prime}}} d \alpha .
$$

By Lemma 4.5 and (4.25),

$$
\mathrm{I}_{1} \leq \frac{C M^{p}}{\sqrt{1+t+a}(1+a-t)^{k-1 / 2}} .
$$

$I_{2}$. From (4.9) and (4.10),

$$
\begin{aligned}
\mathrm{I}_{2} \leq & \frac{C M^{p}}{\sqrt{a}} \int_{a-t}^{t+a} \frac{1}{(1+\alpha)^{p m-\varepsilon^{\prime}}} \int_{\alpha}^{\infty} \frac{\ln (2+\beta)}{(1+\beta)^{(p-1) / 2}} d \beta d \alpha \\
& +\frac{C M^{p}}{\sqrt{a}} \int_{a-t}^{t+a} \frac{1}{\sqrt{a+\alpha-t}(1+\alpha)^{p m-\varepsilon^{\prime}}} \int_{\alpha}^{\infty} \frac{1}{(1+\beta)^{(p-1) / 2}} d \beta d \alpha .
\end{aligned}
$$

This estimate is exactly of the same type as (4.26). Therefore, we obtain

$$
\mathrm{I}_{2} \leq \frac{C M^{p}}{\sqrt{1+t+a}(1+a-t)^{m}} .
$$

$\mathrm{I}_{3}$. We proceed as $\mathrm{II}_{3}$ in Case 1 .

(i) $a>2 t$, or $2 t \geq a \geq t$ and $t \leq 1$. By (4.11) and (4.39), we have

$$
\begin{aligned}
\mathrm{I}_{3} & \leq C M^{p} \int_{t-a}^{a-t} \frac{1}{\sqrt{a-t+\alpha}(1+|\alpha|)^{p m-\varepsilon^{\prime}}} d \alpha \int_{a-t}^{\infty} \frac{1}{\sqrt{t+a+\beta}(1+\beta)^{p / 2-1}} d \beta \\
& \leq \frac{C M^{p}}{(1+a-t)^{(p-3) / 2}} \int_{0}^{a-t}\left(\frac{1}{\sqrt{a-t+\alpha}}+\frac{1}{\sqrt{a-t-\alpha}}\right) \frac{1}{(1+\alpha)^{p m-\varepsilon^{\prime}}} d \alpha .
\end{aligned}
$$


The last estimate is the same as (4.40). Therefore,

$$
\mathrm{I}_{3} \leq \frac{C M^{p}}{\sqrt{1+t+a}(1+a-t)^{m}} .
$$

(ii) $2 t \geq a \geq t$ and $t \geq 1$. From (4.9) and (4.10),

$$
\begin{aligned}
\mathrm{I}_{3} \leq & \frac{C M^{p}}{\sqrt{a}} \int_{t-a}^{a-t} \frac{1}{(1+|\alpha|)^{p m-\varepsilon^{\prime}}} d \alpha \int_{a-t}^{\infty} \frac{\ln (2+\beta)}{(1+\beta)^{(p-1) / 2}} d \beta \\
& +\frac{C M^{p}}{\sqrt{a}} \int_{t-a}^{a-t} \frac{1}{\sqrt{a+\alpha-t}(1+|\alpha|)^{p m-\varepsilon^{\prime}}} d \alpha \int_{a-t}^{\infty} \frac{1}{(1+\beta)^{(p-1) / 2}} d \beta \\
\leq & \frac{C M^{p} \ln (2+a-t)}{\sqrt{1+t+a}(1+a-t)^{(p-3) / 2}} \int_{0}^{a-t} \frac{1}{(1+\alpha)^{p m-\varepsilon^{\prime}}} d \alpha \\
+ & \frac{C M^{p}}{\sqrt{1+t+a}(1+a-t)^{(p-3) / 2}} \\
& \times \int_{0}^{a-t}\left(\frac{1}{\sqrt{a+\alpha-t}}+\frac{1}{\sqrt{a-\alpha-t}}\right) \frac{1}{(1+\alpha)^{p m-\varepsilon^{\prime}}} d \alpha .
\end{aligned}
$$

The last estimate is the same as (4.44). Therefore,

$$
\mathrm{I}_{3} \leq \frac{C M^{p} \ln (2+a-t)}{\sqrt{1+t+a}(1+a-t)^{m}} .
$$

II. We proceed as $\mathrm{II}_{4}$ in Case 1 .

(i) $a \geq 2 t$, or $2 t \geq a \geq t$ and $t \leq 1$. From (4.13) and (4.39),

$$
\begin{aligned}
\mathrm{II} & \leq C M^{p} \int_{-\infty}^{t-a} \frac{1}{\sqrt{t-a-\alpha}(1+|\alpha|)^{p m-\varepsilon^{\prime}}} \int_{-\alpha}^{\infty} \frac{1}{\sqrt{t+a+\beta}(1+\beta)^{p / 2-1}} d \beta d \alpha \\
& \leq C M^{p} \int_{a-t}^{\infty} \frac{1}{\sqrt{t-a+\alpha}(1+\alpha)^{p m+(p-3) / 2-\varepsilon^{\prime}}} d \alpha .
\end{aligned}
$$

The last estimate is similar to (4.47). Using that

$$
\int_{a}^{\infty} \frac{1}{\sqrt{\alpha-b}(1+\alpha)^{k}} d \alpha \leq \frac{C}{(1+a)^{k-1 / 2}} \text { for } k>\frac{1}{2} \text { and } a \geq b \geq 0
$$

and proceeding as in (4.47), (4.48), we obtain

$$
\text { II } \leq \frac{C M^{p}}{\sqrt{1+t+a}(1+a-t)^{m}} .
$$

(ii) $2 t \geq a \geq t$ and $t \geq 1$. From (4.12),

$$
\begin{aligned}
\mathrm{II} \leq & \frac{C M^{p}}{\sqrt{a}} \int_{-\infty}^{t-a} \frac{1}{(1+|\alpha|)^{p m-\varepsilon^{\prime}}} \int_{-\alpha}^{\infty} \frac{\ln (2+\beta)}{(1+\beta)^{(p-1) / 2}} d \beta d \alpha \\
& +\frac{C M^{p}}{\sqrt{a}} \int_{-\infty}^{t-a} \frac{1}{\sqrt{t-a-\alpha}(1+|\alpha|)^{p m-\varepsilon^{\prime}}} \int_{-\alpha}^{\infty} \frac{1}{(1+\beta)^{(p-1) / 2}} d \beta d \alpha \\
\leq & \frac{C M^{p}}{\sqrt{1+t+a}} \int_{a-t}^{\infty} \frac{1}{(1+\alpha)^{p m+(p-3) / 2-\varepsilon^{\prime}-\varepsilon^{\prime \prime}}} d \alpha \\
& +\frac{C M^{p}}{\sqrt{1+t+a}} \int_{a-t}^{\infty} \frac{1}{\sqrt{t-a+\alpha}(1+\alpha)^{p m+(p-3) / 2-\varepsilon^{\prime}}} d \alpha .
\end{aligned}
$$




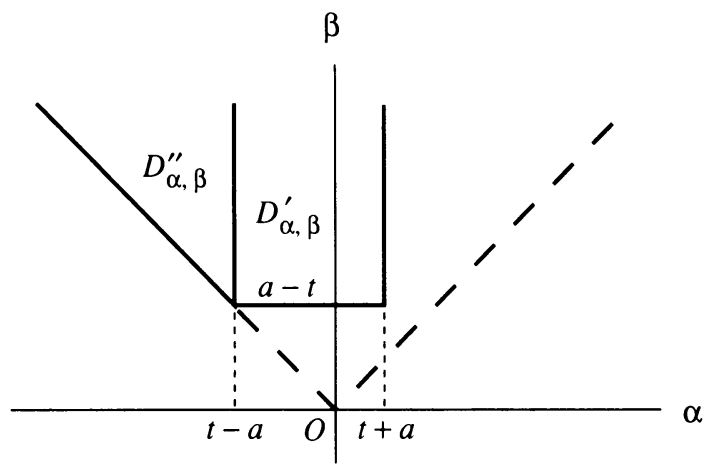

FIGURE 3

The last estimate is similar to (4.49). Proceeding as in (4.49), (4.50), we use (4.57) to obtain

$$
\mathrm{II} \leq \frac{C M^{p}}{\sqrt{1+t+a}(1+a-t)^{m}} .
$$

Case 3. $a \geq|t|$ and $t \leq 0$. As before, changing variables by (4.14), the domains of integration $D^{\prime}$ and $D^{\prime \prime}$ go correspondingly to $D_{\alpha, \beta}^{\prime}$ and $D_{\alpha, \beta}^{\prime \prime}$, where $D_{\alpha, \beta}^{\prime}$ and $D_{\alpha, \beta}^{\prime \prime}$ are pictured in Figure 3.

I. From (4.11) and by (4.39), we have

$$
\begin{aligned}
\mathrm{I} \leq C M^{p} \int_{t-a}^{t+a} \frac{1}{\sqrt{a-t+\alpha}(1+|\alpha|)^{p m-\varepsilon^{\prime}}} d \alpha \int_{a-t}^{\infty} \frac{1}{\sqrt{t+a+\beta}(1+\beta)^{p / 2-1}} d \beta \\
\leq \frac{C M^{p}}{(1+a-t)^{(p-3) / 2}}\left(\int_{0}^{t+a} \frac{1}{\sqrt{a-t+\alpha}(1+\alpha)^{p m-\varepsilon^{\prime}}} d \alpha\right. \\
\left.\quad \quad \quad \int_{0}^{a-t} \frac{1}{\sqrt{a-t-\alpha}(1+\alpha)^{p m-\varepsilon^{\prime}}} d \alpha\right) \\
\leq \frac{C M^{p}}{(1+a+|t|)^{(p-3) / 2}} \int_{0}^{a+|t|}\left(\frac{1}{\sqrt{a+|t|+\alpha}}+\frac{1}{\sqrt{a+|t|-\alpha}}\right) \frac{1}{(1+\alpha)^{p m-\varepsilon^{\prime}}} d \alpha .
\end{aligned}
$$

The last estimate is the same as (4.40). Therefore,

$$
\mathrm{I} \leq \frac{C M^{p}}{(1+a+|t|)^{m+1 / 2}} \leq \frac{C M^{p}}{\sqrt{1+a+|t|}(1+|a-| t||)^{m}} .
$$

II. From (4.13) and (4.39),

$$
\begin{aligned}
\mathrm{II} & \leq C M^{p} \int_{-\infty}^{t-a} \frac{1}{\sqrt{t-a-\alpha}(1+|\alpha|)^{p m-\varepsilon^{\prime}}} \int_{-\alpha}^{\infty} \frac{1}{\sqrt{t+a+\beta}(1+\beta)^{p / 2-1}} d \beta d \alpha \\
& \leq C M^{p} \int_{a-t}^{\infty} \frac{1}{\sqrt{t-a+\alpha}(1+\alpha)^{p m+(p-3) / 2-\varepsilon^{\prime}}} d \alpha \\
& =C M^{p} \int_{a+|t|}^{\infty} \frac{1}{\sqrt{\alpha-a-|t|}(1+\alpha)^{p m+(p-3) / 2-\varepsilon^{\prime}}} d \alpha .
\end{aligned}
$$

Using (4.57), we obtain

$$
\mathrm{II} \leq \frac{C M^{p}}{(1+a+|t|)^{m+1 / 2}} \leq \frac{C M^{p}}{\sqrt{1+a+|t|}(1+|a-| t||)^{m}} .
$$




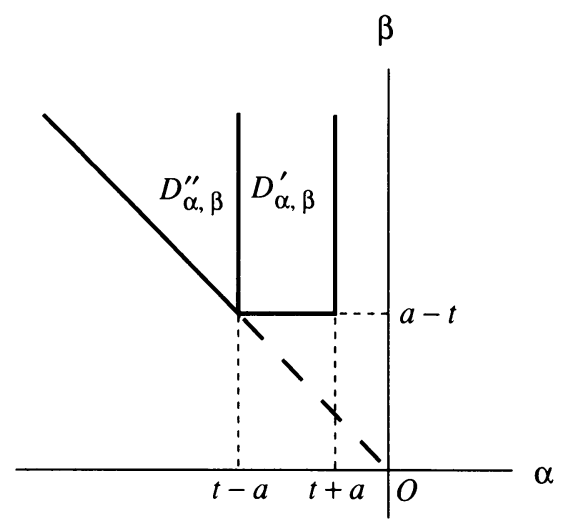

FIGURE 4

Case 4. $|t| \geq a$ and $t \leq 0$. As before, changing variables by (4.14), the domains of integration $D^{\prime}$ and $D^{\prime \prime}$ go to $D_{\alpha, \beta}^{\prime}$ and $D_{\alpha, \beta}^{\prime \prime}$ respectively, where $D_{\alpha, \beta}^{\prime}$ and $D_{\alpha, \beta}^{\prime \prime}$ are shown in Figure 4.

I. Proceeding as part I of Case 3, we use (4.57) to obtain (4.62)

$$
\begin{aligned}
\mathrm{I} & \leq C M^{p} \int_{t-a}^{t+a} \frac{1}{\sqrt{a-t+\alpha}(1+|\alpha|)^{p m-\varepsilon^{\prime}}} d \alpha \int_{a-t}^{\infty} \frac{1}{\sqrt{t+a+\beta}(1+\beta)^{p / 2-1}} d \beta \\
& \leq \frac{C M^{p}}{(1+a+|t|)^{(p-3) / 2}} \int_{|t|-a}^{a+|t|} \frac{1}{\sqrt{a+|t|-\alpha}(1+\alpha)^{p m-\varepsilon^{\prime}}} d \alpha \\
& \leq \frac{C M^{p}}{\sqrt{1+a+|t|}(1+|a-| t||)^{m}} .
\end{aligned}
$$

II. Using (4.57), we can estimate II in the same manner as II in Case 3. Therefore,

$$
\mathrm{II} \leq \frac{C M^{p}}{\sqrt{1+a+|t|}(1+|a-| t||)^{m}} .
$$

This completes the proof of Lemma 4.1.

The basic estimate for $0<k \leq \frac{1}{2}$ is the following lemma which we can prove in the same manner as Lemma 4.1 .

Lemma 4.7. Assume that $u(x, t) \in C\left(R^{2} \times R\right)$ satisfies

$$
|u(x, t)| \leq \begin{cases}\frac{M}{\sqrt{1+|t|+a}}\left(1+\ln \frac{1+|t|+a}{1+|| t|-a|}\right) & \left(k=\frac{1}{2}\right) \\ \frac{M}{(1+|t|+a)^{k}} & \left(0<k<\frac{1}{2}\right)\end{cases}
$$

where $a=|x|$.

Let $k>2 /(p-1)$. Then, there exists a constant $C_{p, k}$ depending only on $p$ and $k$, not on $M$ such that

$$
\left.|L| u\right|^{p}(x, t) \mid \leq \frac{C_{p, k} M^{p}}{(1+|t|+a)^{k}} \quad\left(0<k \leq \frac{1}{2}\right) .
$$


Remark 4.8. Note that if $0<k \leq \frac{1}{2}$ and $k>2 /(p-1)$, then $p>5$. Thus, the proof of this lemma is easier than that of Lemma 4.1.

\section{Proof of Theorem 2.2}

In this section, we prove Theorem 2.2 and Corollary 2.4 .

Proof of part (i) of Theorem 2.2.

Part 1 (Proof of the existence). From (2.7), we have

$$
\begin{gathered}
|u(x, t)| \leq \begin{cases}\|u\|_{k} & \left(0<k<\frac{1}{2}, \quad \frac{1}{2}<k<1\right), \\
2\|u\|_{k} & \left(k=\frac{1}{2}\right),\end{cases} \\
\left\||u|^{\theta}|v|^{1-\theta}\right\|_{k} \leq\|u\|_{k}^{\theta}\|v\|_{k}^{1-\theta} \quad \text { for } 0 \leq \theta \leq 1,
\end{gathered}
$$

and by Lemmas 4.1 and 4.7 ,

$$
\begin{aligned}
\left\|L|u|^{p}\right\|_{k} & \leq C_{p, k}\|u\|_{k}^{p}, \\
\left\|L|u|^{\theta p}|v|^{(1-\theta) p}\right\|_{k} & \leq C_{p, k}\|u\|_{k}^{\theta p}\|v\|_{k}^{(1-\theta) p},
\end{aligned}
$$

where $C_{p, k}$ a constant given in Lemmas 4.1 and 4.7 .

We can verify easily that the linear space $X_{k}$ defined by (2.5) is complete with respect to the norm (2.6) and (2.7).

We denote by $X_{0}$ the closed subset of $X_{k}$ given by

$$
X_{0}=\left\{u \in X_{k}:\|u\|_{X_{k}} \leq 2 B_{k} C_{k} \varepsilon\right\},
$$

where $C_{k}$ is a constant in (3.1) and

$$
B_{k}= \begin{cases}1 / \ln 2 & \left(k>1, \frac{1}{2}<k<1\right), \\ 1 & \left(k=1,0<k \leq \frac{1}{2}\right) .\end{cases}
$$

Then, it follows from Lemma 3.1 that

$$
\left\|u_{0}^{-}\right\|_{X_{k}} \leq B_{k} C_{k} \varepsilon
$$

Hence, $u_{0}^{-} \in X_{0}$. Now we define the map $T$ by

$$
T u=u_{0}^{-}+L F(u) \text {. }
$$

We can derive (see $[7,20])$

$$
\begin{aligned}
\|L F(u)\|_{k} & \leq C_{p, k} \lambda\left(2 B_{k} C_{k} \varepsilon\right)^{p}, \\
\left\|\mathrm{D}_{j} L F(u)\right\|_{k} & \leq 2 C_{p, k} \lambda\left(2 B_{k} C_{k} \varepsilon\right)^{p}, \\
\left\|\mathrm{D}_{i} \mathrm{D}_{j} L F(u)\right\|_{k} & \leq 5 C_{p, k} \lambda\left(2 B_{k} C_{k} \varepsilon\right)^{p},
\end{aligned}
$$

and

$$
\begin{aligned}
\|T u-T v\|_{k} & \leq C_{p, k} \lambda\left(2 B_{k} C_{k} \varepsilon\right)^{p-1}\|u-v\|_{X_{k}}, \\
\left\|\mathrm{D}_{j}(T u-T v)\right\|_{k} & \leq 2 C_{p, k} \lambda\left(2 B_{k} C_{k} \varepsilon\right)^{p-1}\|u-v\|_{X_{k}}, \\
\left\|\mathrm{D}_{i} \mathrm{D}_{j}(T u-T v)\right\|_{k} & \leq 5 C_{p, k} \lambda\left(2 B_{k} C_{k} \varepsilon\right)^{p-1}\|u-v\|_{X_{k}}
\end{aligned}
$$

for $u, v \in X_{0}$, where $\mathrm{D}_{j}=\partial / \partial x_{j} \quad(j=1,2)$.

We see that

$$
\begin{gathered}
\|T u\|_{X_{k}} \leq B_{k} C_{k} \varepsilon+20 C_{p, k} \lambda\left(2 B_{k} C_{k} \varepsilon\right)^{p} \leq 2 B_{k} C_{k} \varepsilon, \\
\|T u-T v\|_{X_{k}} \leq 20 C_{p, k} \lambda\left(2 B_{k} C_{k} \varepsilon\right)^{p-1}\|u-v\|_{X_{k}} \leq \frac{1}{2}\|u-v\|_{X_{k}}
\end{gathered}
$$


for $u, v \in X_{0}$, provided

$$
20 C_{p, k} \lambda 2^{p}\left(B_{k} C_{k} \varepsilon\right)^{p-1} \leq 1 .
$$

Thus, if we choose $\varepsilon>0$ so small that (5.1) holds, then $T$ is a contraction of $X_{0}$ into itself, and so $T$ admits a unique fixed point $u \in X_{0}$, which satisfies (2.4).

Part 2 (Proof of (2.8)). To prove (2.8), as is well known we have only to show that

$$
\int_{-\infty}^{t}\|F(u(s))\|_{L^{2}\left(R^{2}\right)} d s \leq \frac{C}{(1+|t|)^{l}} \rightarrow 0 \quad(t \rightarrow-\infty),
$$

where $l$ is given by (2.9).

We split the integral in (5.2) into five parts, following Pecher [16] and use the decay estimate of $u$ from $u \in X_{k}$.

First we shall prove (5.2) for $k>\frac{1}{2}$.

(1)

$$
\begin{aligned}
\int_{-\infty}^{t} & \left(\int_{|x| \leq|s| / 2}|F(u(x, s))|^{2} d x\right)^{1 / 2} d s \\
& \leq C \int_{-\infty}^{t} \frac{1}{(1+|s|)^{p(m+1 / 2)-\varepsilon^{\prime}}}\left(\int_{|x| \leq|s| / 2} d x\right)^{1 / 2} d s \\
& \leq C \int_{-\infty}^{t} \frac{1}{(1+|s|)^{p(m+1 / 2)-1-\varepsilon^{\prime}}} d s \\
& \leq \frac{1}{(1+|t|)^{p(m+1 / 2)-2-\varepsilon^{\prime}}} \leq \frac{C}{(1+|t|)^{m}} .
\end{aligned}
$$

We have used (4.17), (4.18), and (4.25) in the last inequality.

(2)

$$
\begin{aligned}
\int_{-\infty}^{t} & \left(\int_{|s| / 2 \leq|x| \leq|s|-1}|F(u(x, s))|^{2} d x\right)^{1 / 2} d s \\
& \leq C \int_{-\infty}^{t} \frac{1}{(1+|s|)^{p / 2}}\left(\int_{|s| / 2}^{|s|-1} \frac{r}{(1+|r-| s||)^{2 p m-2 \varepsilon^{\prime}}} d r\right)^{1 / 2} d s \\
& \leq C \int_{-\infty}^{t} \frac{|s|^{1 / 2}}{(1+|s|)^{p / 2}}\left(\int_{|s| / 2}^{|s|-1} \frac{1}{(1+|s|-r)^{2 p m-2 \varepsilon^{\prime}}} d r\right)^{1 / 2} d s \\
& \equiv \mathrm{I} .
\end{aligned}
$$

We need to consider two cases.

(a) $m=\frac{1}{2},(p-3) / 2$, or $m=k-\frac{1}{2}$ and $2 p\left(k-\frac{1}{2}\right)>1$. Using (4.17), (4.18) and (4.19),

$$
\mathrm{I} \leq C \int_{-\infty}^{t} \frac{1}{(1+|s|)^{(p-1) / 2}} d s \leq \frac{C}{(1+|t|)^{(p-3) / 2}} \leq \frac{C}{(1+|t|)^{m}} .
$$

(b) $m=k-\frac{1}{2}$ and $2 p\left(k-\frac{1}{2}\right) \leq 1$. By (4.25),

$$
\mathrm{I} \leq C \int_{-\infty}^{t} \frac{1}{(1+|s|)^{k+1}} d s \leq \frac{C}{(1+|t|)^{k}} .
$$


(3)

$$
\begin{aligned}
& \int_{-\infty}^{t}\left(\int_{|s|-1 \leq|x| \leq|s|+1}|F(u(x, s))|^{2} d x\right)^{1 / 2} d s \\
& \quad \leq C \int_{-\infty}^{t} \frac{1}{(1+|s|)^{p / 2}}\left(\int_{|s|-1 \leq|x| \leq|s|+1} d x\right)^{1 / 2} d s \\
& \quad \leq C \int_{-\infty}^{t} \frac{1}{(1+|s|)^{(p-1) / 2}} d s \leq \frac{C}{(1+|t|)^{m}} .
\end{aligned}
$$

(4) The integral over $|s|+1 \leq|x| \leq 2|s|$ can be estimated in the same manner as that in (2).

(5)

$$
\begin{aligned}
& \int_{-\infty}^{t}\left(\int_{|x| \geq 2|s|}|F(u(x, s))|^{2} d x\right)^{1 / 2} d s \\
& \quad \leq C \int_{-\infty}^{t}\left(\int_{|x| \geq 2|s|} \frac{1}{(1+|x|)^{2 p(m+1 / 2)-2 \varepsilon^{\prime}}} d x\right)^{1 / 2} d s \\
& \quad \leq C \int_{-\infty}^{t}\left(\int_{2|s|}^{\infty} \frac{r}{(1+r)^{2 p(m+1 / 2)-2 \varepsilon^{\prime}}} d r\right)^{1 / 2} d s \\
& \quad \leq C \int_{-\infty}^{t} \frac{1}{(1+|s|)^{p(m+1 / 2)-1-\varepsilon^{\prime}}} d s \leq \frac{C}{(1+|t|)^{m}} .
\end{aligned}
$$

Similarly, we can prove (5.2) for $0<k \leq \frac{1}{2}$.

Proof of part (ii) of Theorem 2.2. We define

$$
u_{0}^{+}(x, t)=u(x, t)-\frac{1}{2 \pi} \int_{t}^{\infty} \int_{|x-y| \leq s-t} \frac{F(u(y, s))}{\sqrt{(s-t)^{2}-|x-y|^{2}}} d y d s .
$$

Then, clearly $u_{0}^{+}$is a $C^{2}$-solution of $(2.2)$ and as before, we can show that

$$
\left\|u(t)-u_{0}^{+}(t)\right\|_{e} \leq \frac{C}{(1+|t|)^{l}} \rightarrow 0 \quad(t \rightarrow+\infty) .
$$

This completes the proof of Theorem 2.2.

Proof of Corollary 2.4. From (2.12), (H1), and the decay estimate of $u$,

$$
\begin{aligned}
\left|\int_{R^{2}} G(u(t)) d x\right| & \leq C \int_{R^{2}}|u|^{p+1}(t) d x \\
& \leq C \int_{R^{2}} \frac{1}{(1+|x|+|t|)^{(p+1) / 2}(1+|| x|-| t||)^{m(p+1)-\varepsilon^{\prime}}} d x \\
& \leq C \int_{0}^{\infty} \frac{r}{(1+r+|t|)^{(p+1) / 2}} d r \\
& \leq \frac{C}{(1+|t|)^{(p-3) / 2}} \rightarrow 0 \quad(t \rightarrow \pm \infty),
\end{aligned}
$$

if $k>\frac{1}{2}$. 
Similarly, if $0<k \leq \frac{1}{2}$,

$$
\left|\int_{R^{2}} G(u(t)) d x\right| \leq \frac{C}{(1+|t|)^{k}} \rightarrow 0 \quad(t \rightarrow \pm \infty) .
$$

We also have

$$
\left\|u_{0}^{-}(0)\right\|_{e}^{2}=\left\|u_{0}^{-}(t)\right\|_{e}^{2}, \quad\left\|u_{0}^{+}(0)\right\|_{e}^{2}=\left\|u_{0}^{+}(t)\right\|_{e}^{2} .
$$

Therefore, (2.11) follows from (2.8), (2.10), (5.3), (5.4), (5.5) and the energy conservation law of $(2.1)$ :

$$
\frac{1}{2}\|u(t)\|_{e}^{2}+\int_{R^{2}} G(u(t)) d x=\frac{1}{2}\|u(s)\|_{e}^{2}+\int_{R^{2}} G(u(s)) d x, \quad t, s \in R .
$$

This completes the proof of Corollary 2.4 .

\section{ACKNOWLEDGMENTS}

The author would like to express his gratitude to Professor Yoshio Tsutsumi, who suggested the problem, for his stimulating discussions and valuable advice. It is also a pleasure to acknowledge Professor Takao Kakita for his helpful advice and constant encouragement.

Added in proof. Recently, K. Kubota and K. Mochizuki (On small data scattering for 2-dimensional semilinear wave equations, Hokkaido Math. J. 22 (1993), 79-97) have obtained independently a similar result using different methods from ours. However our decay estimates of solutions of (2.1) are slightly sharper than theirs.

\section{REFERENCES}

1. R. Agemi and $\mathbf{H}$. Takamura, The lifespan of classical solutions to nonlinear wave equations in two space dimensions, Hokkaido Math. J. 21 (1992), 517-542.

2. F. Asakura, Existence of a global solution to a semi-linear wave equation with slowly decreasing initial data in three space dimensions, Comm. Partial Differential Equations 11 (1986), 1459-1487.

3. J. Ginibre and G. Velo, Conformal invariance and time decay for non linear wave equations. I, Ann. Inst. H. Poincaré Phys. Théor. 47 (1987), 221-261.

4. __ Conformal invariance and time decay for non linear wave equations. II, Ann. Inst. H. Poincaré Phys. Théor. 47 (1987), 263-276.

5. _ Scattering theory in the energy space for a class of non-linear wave equations, Comm. Math. Phys. 123 (1989), 535-573.

6. R. T. Glassey, Finite-time blow-up for solutions of nonlinear wave equations, Math. Z. 177 (1981), 323-340.

7. __, Existence in the large for $\square u=F(u)$ in two space dimensions, Math. Z. 178 (1981), 233-261.

8. F. John, Blow-up of solutions of nonlinear wave equations in three space dimensions, Manuscripta Math. 28 (1979), 235-268.

9. __ Nonlinear wave equations, formation of singularities, Univ. Lecture Ser., Amer. Math. Soc., Providence, RI, 1990.

10. S. Klainerman, Long-time behavior of solutions to nonlinear evolution equations, Arch. Rational Mech. Anal. 78 (1982), 73-98.

11. M. Kovalyov, Long-time behaviour of solutions of a system of nonlinear wave equations, Comm. Partial Differential Equations 12 (1987), 471-501. 
12. __ Long-time existence of solutions of nonlinear wave equations, Ph.D. thesis, Courant Institute of Mathematical Sciences, New York University, 1986.

13. K. Kubota, Existence of a global solution to a semi-linear wave equation with initial data of non-compact support in low space dimensions, Hokkaido Math. J. 22 (1993), 123-180.

14. K. Mochizuki and T. Motai, The scattering theory for the nonlinear wave equation with small data, J. Math. Kyoto Univ. 25 (1985), 703-715.

15. __ The scattering theory for the nonlinear wave equation with small data. II, Publ. Res. Inst. Math. Sci. 23 (1987), 771-790.

16. H. Pecher, Scattering for semilinear wave equations with small data in three space dimensions, Math. Z. 198 (1988), 277-289.

17. __ Global smooth solutions to a class of semilinear wave equations with strong nonlinearities, Manuscripta Math. 69 (1990), 71-92.

18. J. Schaeffer, The equation $u_{t t}-\Delta u=|u|^{p}$ for the critical value of $p$, Proc. Roy. Soc. Edinburgh Sect. A 101 (1985), 31-44.

19. W. A. Strauss, Nonlinear scattering theory at low energy, J. Funct. Anal. 41 (1981), 110-133.

20. K. Tsutaya, Global existence theorem for semilinear wave equations with non-compact data in two space dimensions, J. Differential Equations 104 (1993), 332-360.

21. __ Global existence and the life span of solutions of semilinear wave equations with data of non compact support in three space dimensions, (to appear).

22. __ A global existence theorem for semilinear wave equations with data of non compact support in two space dimensions, Comm. Partial Differential Equations 17 (1992), 19251954.

Department of Mathematics, School of Science and Engineering, Waseda University, 3-4-1 OhKubo, Shinjuku, Tokyo 169, Japan

Current address: Department of Mathematics, Hokkaido University, Sapporo 060, Japan

E-mail address: tsutaya@math.hokudai.ac.jp 